\title{
TRATTAMENTO LVRC: NUOVO IMAGING INTERVENTISTICO PER IL TRATTAMENTO DELL'ENFISEMA POLMONARE
}

\author{
Dott. Erika Vallefuoco ${ }^{1}$, Dott. Andrea Caldeo ${ }^{2}$ \\ ${ }^{1}$ T.S.R.M presso Istituto di Ricerca Diagnostica e Nucleare SDN \\ ${ }^{2}$ T.S.R.M presso Università di Zurigo
}

KEYWORDS: Lung Volume Reduction, Coil, Polmunary Emphysema, Enfisema Polmonare

\section{ABSTRACT}

\begin{abstract}
Background Treatment of pulmonary emphysema with Lung Volume Reduction Coil (LVRC) technique and related thoracic imaging preoperative, interventional, under radioscopic guidance, and postoperative.

For the intervention, Nitinol coils are used, implanted through a catheter under radioscopic guidance using an Intensifier. The treatment is performed with induction of anesthesia, during one or two sessions.

Coils are implanted on patients in different lobars regions, depending on the case. The patient's status is monitored in 3 phases: Phase 1 Preoperative; Phase 2 Intervention; Phase 3 Post-operative up to the following 5 years. This technique, given the effectiveness and an almost total absence of side effects, turns out to be a valid alternative to others methods for the treatment of severe pulmonary emphysema.

Through the analysis of the monitoring results, it is possible to infer that the patients subjected to LVRC (Coil) have high clinical benefits, but still not accompanied by significant long-term functional improvements.
\end{abstract}

\section{INTRODUZIONE}

L'Enfisema Polmonare è una patologia ostruttiva causata dalla degenerazione degli alveoli polmonari, causando la perdita dell'elasticità parenchimale con conseguente dilatazione degli spazi aerei a valle dei bronchioli terminali e perdita delle loro pareti con assenza di fibrosi. Le sue conseguenze sono la progressiva dispnea, tosse e un evidente rallentamento dell'espirazione (FEV) dovuta ad un ristagno dell'aria. In caso di Paziente con Enfisema Severo, è possibile ricorrere ad un intervento di "Lung Volume Reduction Coil (LVRC)", che consente un recupero dell'elasticità polmonare e migliorando la funzione meccanica respiratoria.

\section{MATERIALI E METODI}

L'intervento è indicato nei pazienti con enfisema di grado severo. Tale condizione è indicata dal coefficiente di Tiffaneau, ovvero (FEV1/FVC); in cui con FEV1 (Forced Expiratory Volume in the 1st second) si indica il Volume Espiratorio Massimo al Primo secondo, mentre con FVC (Forced Vital Capacity) si indica la Capacità Vitale Forzata. Quando tale fattore risulta: (FEV1/FVC) $\leq 70 \%$ e FEV1 $<40 \%$, l'enfisema si definirà di grado Severo, condizione necessaria per essere sottoposti all'intervento. Per l'esecuzione dell'intervento non è richiesta alcuna preparazione particolare da parte del paziente. E' sufficiente mantenere il digiuno dalla sera precedente e non assumere liquidi nelle 6 ore precedenti all'intervento.

Quest'ultimo viene eseguito su Paziente in anestesia generale, attraverso un catetere bronchiale sotto guida radioscopica.

Vengono utilizzati dispositivi a spirale (Coil) in Niti-

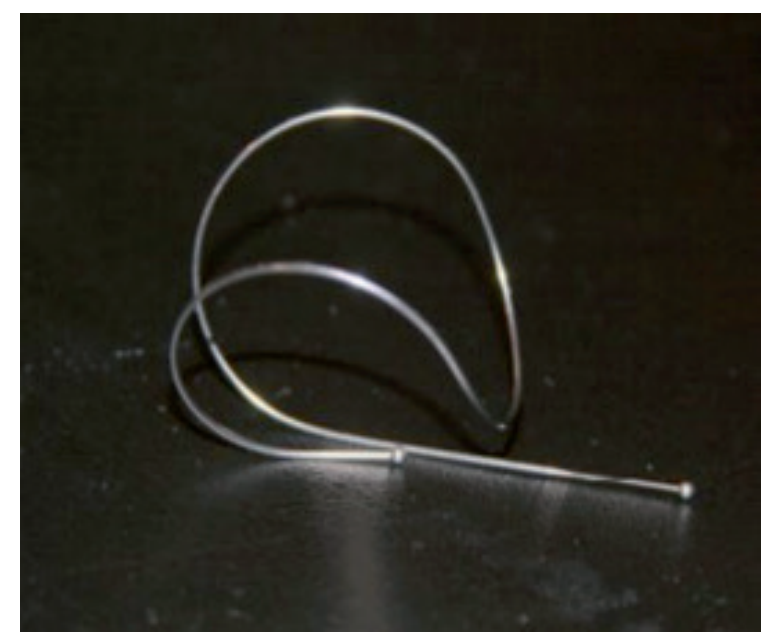

Figura 1 - Foto Coil.

nol, una lega di nichel e titanio biocompatibile, superelastico, duttile ed amagnetico.

Queste Coil vengono posizionate attraverso il catetere nelle vie aeree, ove, assumendo la loro forma originaria, determinano così una contrazione e un accartocciamento del parenchima polmonare adiacente.

\section{TECNICA}

In Radioscopia, una guida raggiunge l'area desiderata. Successivamente viene posizionato distalmente un catetere a ca. $15 \mathrm{~mm}$ dalla pleura. A questo punto viene misurata la lunghezza della via aerea per determinare la lunghezza della Coil, compresa tra i 10 e i 20 $\mathrm{cm}$, che viene inserita in forma rettilinea (compressa) dopo la rimozione della guida, monitorando l'operazione in Radioscopia. Rilasciata la Coil, quest'ultima 


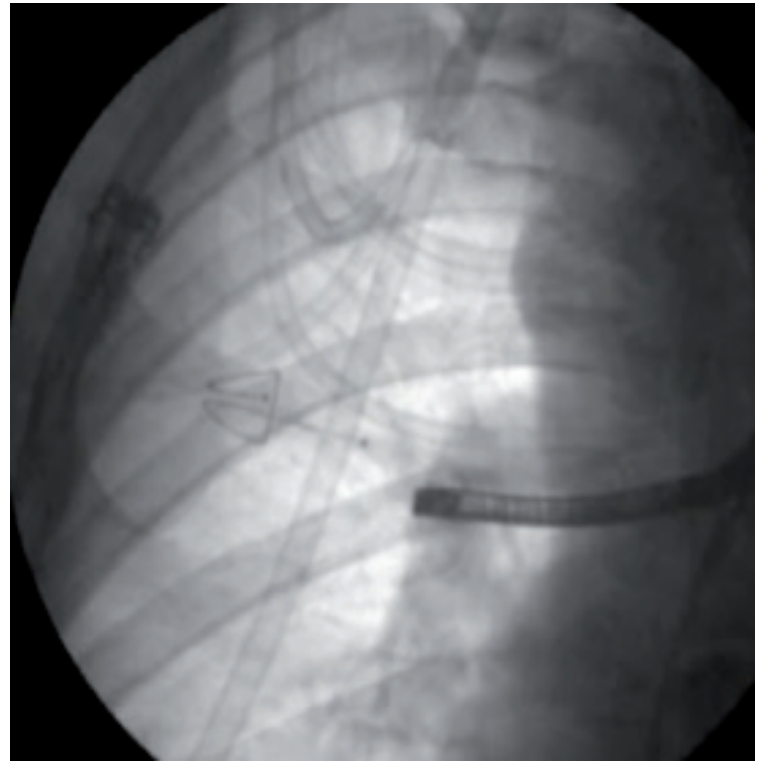

Figura 2 - Inserimento della Coil.

assume nuovamente la sua forma originale a spirale, ritraendosi, e determinando così una contrazione del parenchima enfisematoso. A questo punto, il catetere viene rimosso, lasciando la Coil all'interno del parenchima.

Durante una singola seduta è possibile impiantare fino a 10 Coil, anche bilateralmente, a seconda della gravità del caso. In media il trattamento ha una durata di 30 minuti circa.
L'esecuzione dell'intervento in una regione anatomica con elevata risoluzione di contrasto naturale quale il Torace, consente di poter monitorare l'intero intervento mantenendo bassissimi livelli di dose. I controlli successivi all'intervento dovranno essere effettuati in proiezioni Standard in massima espirazione per accentuare il naturale contrasto parenchimale.

\section{RISULTATI}

I dati rilevati dallo studio effettuato sui pazienti trattati con Coil, mostrano l'assenza di eventi avversi rilevanti, in particolare di pneumotorace. In alcuni casi si è registrata la comparsa di riacutizzazioni bronchiali nella fase immediatamente successiva all'impianto delle Coil, in tutti i casi risolte favorevolmente con terapia farmacologica. Tuttavia, anche questa procedura determina un miglioramento generale, ma non arresta il decorso della patologia, evidenziando quindi una quasi totale perdita di risposta al trattamento al controllo a 12 mesi. La tabella mostra 1'andamento del FEV1 prima della procedura, dopo 3 mesi, 6 mesi e 12 mesi dall'intervento.

\section{DISCUSSIONE}

Lo studio è stato effettuato in seguito all'esecuzione di un esame Rx Torace di controllo presso il "Radiologisches Zentrum Schwarzwald-Baar" di VillingenSchwenningen in Germania. A seguito di tale esame, la presenza di Coil all'interno del parenchima polmonare ci ha spinti ad effettuare ricerche sull'intervento e sulle strutture ove tale veniva eseguito. Tra

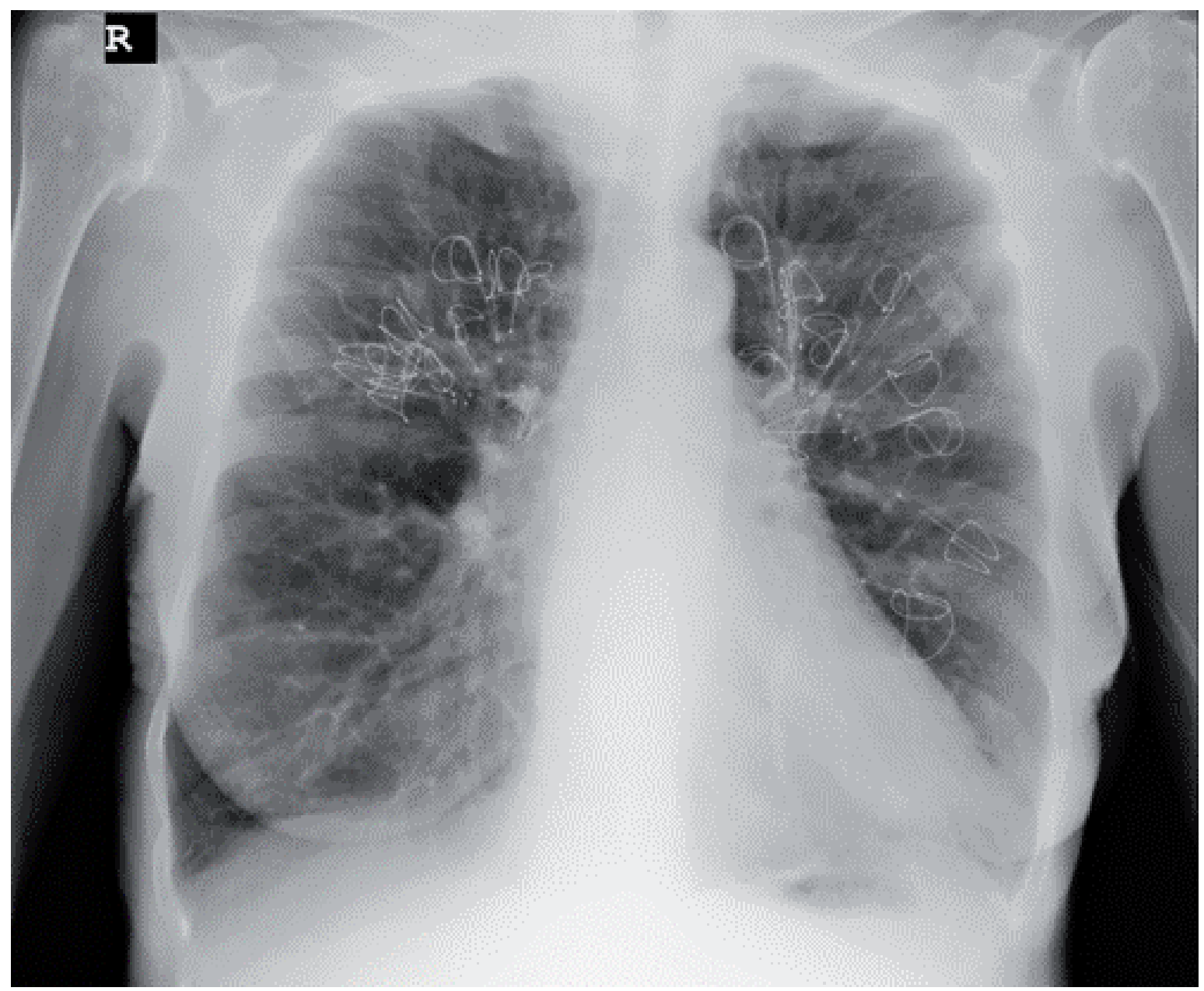

Figura 4 - Controllo dopo 6 mesi dall'intervento[/dkpdf-remove] . 


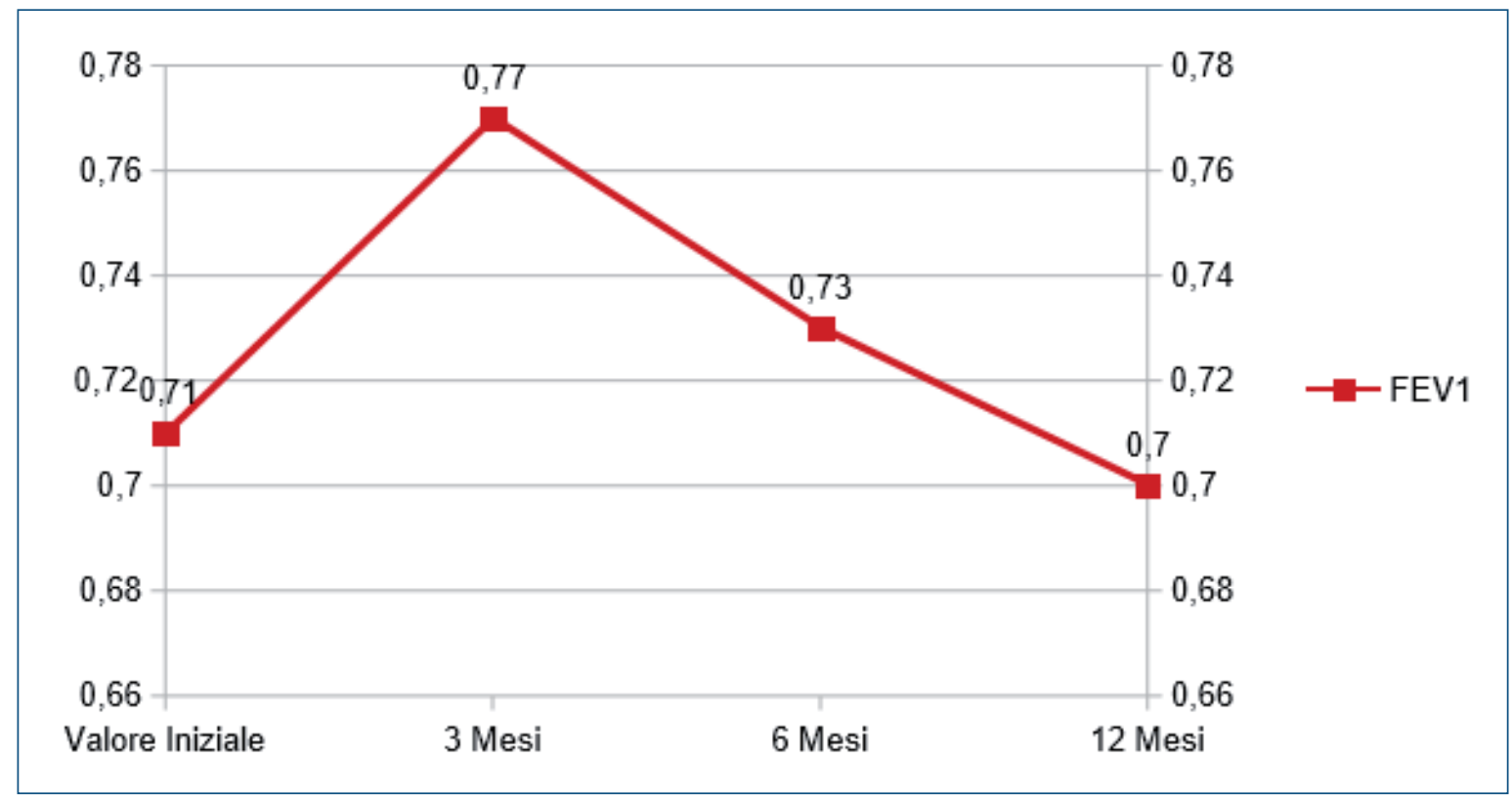

Figura 4 - Andamento del FEV1 nelle fasi pre- e post-operatorie.

questi abbiamo individuato il "Krankenhaus vom Roten Kreuz" sito in Stoccarda, ed il "Pneumologische Lehrklinik Universitätsmedizin” di Göttingen. Sulla base dei dati raccolti da queste strutture e da studi effettuati in Italia ed in Ohio, abbiamo impostato la nostra ricerca che ci ha permesso di determinare la tecnica di esecuzione di tale intervento ed il ruolo del TSRM nelle fasi diagnostiche, d'intervento e postoperatorie.

\section{CONCLUSIONI}

Dallo studio si evince che la tecnica Coil risulta un tipo di intervento i cui risultati generano ottimi benefici dal punto di vista clinico, ma non sono stati riscontrati significativi miglioramenti funzionali in confronto ad altre tecniche attualmente utilizzate per il trattamento dell'enfisema polmonare. Ciononostante, tale tecnica rappresenta un nuovo tipo di Imaging diagnostico ed interventistico di cui è utile essere a conoscenza.

\section{REFERENCES}

1. Fishman A, Martinez F, Naunheim K - A randomized trial comparing lung-volume-reduction surgery with medical therapy for severe emphysema. N Engl J Med 2003;348:2059-73.

2. Venuta F, Diso D, Anile M - Bronchoscopic lung volume reduction as a bridge to lung transplantation in patients with chronic obstructive pulmonary disease. Eur J Cardiothorac Surg 2011; 39: 364-367.

3. Shah PL, Zoumot Z, Singh S - Endobronchial coils for the treatment of severe emphysema with hyperinflation (RESET): a randomised controlled trial. Lancet Respir Med 2013; 1: 233-240.

4. Deslee G, Klooster K, Hetzel M - Lung volume reduction coil treatment for patients with severe emphysema: a European multicentre trial. Thorax 2014; 69: 980-986.

5. Slebos D-J, Klooster K, Ernst A - Bronchoscopic lung volume reduction coil treatment of patients with severe heterogeneous emphysema. Chest 2012; 142: 574-582.

6. Toma TP, Hopkinson NS, Hillier J - Bronchoscopic volume reduction with valve implants in patients with severe emphysema. Lancet 2003;361:931-3. 